\title{
Acute tadalafil administration increases plasma fatty acids without changes in the inflammatory response in healthy men
}

\author{
Roberta Ceci ${ }^{{ }^{\star *}}$, Guglielmo Duranti ${ }^{*}$, Paolo Sgrò², Stefania Sabatini and Luigi Di Luigi² \\ 1 Università degli Studi di Roma "Foro Italico", Department of Movement, Human and Health Sciences, Unit of Biology, Genetics and Biochem- \\ istry, Rome, Italy; 2Università degli Studi di Roma "Foro Italico", Department of Movement, Human and Health Sciences, Unit of Endocrinology, \\ Rome, Italy
}

Purpose: Tadalafil, the phosphodiesterase type 5 inhibitor (PDE5I), has been shown to reduce visceral adipose tissue in rabbit and to improve lean mass content in non-obese men. In order to clarify this effect in humans, in the present study we determined the impact of an acute oral tadalafil administration on lipolysis by evaluating plasma free fatty acids (FFAs) and glycerol. FFAs are potential modulator of inflammation response that we evaluated through tumor necrosis factor alpha (TNFa), interleukin 6 (IL6), interleukin 8 (IL8) and interleukin 10 (IL10) plasma levels. Moreover, we determined whether the effects of tadalafil would be reflected in variation of plasma levels of CGMP and NO, two important molecules involved in PDE5Is signaling. Methods: Twelve healthy subjects were supplemented with $20 \mathrm{mg}$ of tadalafil or a placebo, in a double-blind, randomized, cross-over design. Blood samples were collected immediately before, and at 2, 6, and 24 hours post ingestion, and assayed for biochemical analysis. Results: A condition effect was noted for FFAs and glycerol, with values higher for tadalafil when compared to the placebo group, at 2 and 6 hours post ingestion. No statistically significant effects were noted for glucose, cGMP, nitrate and nitrite. No inflammatory response was induced by tadalafil. Conclusion: Tadalafil, in human subjects, increases lipolysis as evidenced by a significant increase in circulating FFAs and glycerol, without affecting the plasma cGMP and NO levels; noticeably, the increase in FFAs did not develop an inflammatory response. Further wellcontrolled studies are warranted to assess the impact of tadalafil administration on weight/fat loss.

Key words: tadalafil, lipolysis, FFAs, cytokines, cGMP

Received: 28 July, 2017; revised: 26 October, 2017; accepted: 31 October, 2017; available on-line: 05 December, 2017

*These authors contributed equally to this work e-mail: roberta.ceci@uniroma4.it

Abbreviations: PDE5Is, phosphodiesterase type 5 inhibitors; CGMP, cyclic guanosine monophoshpate; FFAs, free fatty acids; Gly, glycerol; Glu, glucose; NO, nitric oxide; IL6, interleukin 6; IL8, interleukin 8; IL10, interleukin 10; TNFa, tumor necrosis factor alpha

\section{INTRODUCTION}

Phosphodiesterase type 5 inhibitors (PDE5Is), largely used to treat erectile dysfunction (ED) (Corbin \& Francis, 2002) and for recreational purposes, such as sports supplements (Di Luigi, 2008), act by extending the action of cyclic guanosine monophosphate (cGMP) (Aversa, 2010), a second messenger responsible for many biological processes (Beavo \& Brunton, 2002). cGMP is one of the intracellular signal transduction mediators of nitric oxide (NO), a molecule with a major role in energy substrate metabolism; indeed a decrease in NO levels, with consequently reduced cGMP production, impairs muscle glucose uptake (Dai et al., 2013). PDE5 inhibition enhances the cGMP-dependent endocrine/metabolic effects of NO by increasing and/or maintaining the intracellular levels of NO-induced cGMP. In men with ED, PDE5Is administration seems to exert positive effects on metabolism, counteracting insulin resistance through possible interactions with glucose homeostasis (Armani et al., 2011).

Among the PDE5Is used for ED, tadalafil exhibits some differences in pharmacokinetic properties, interactions with food and alcohol, half-life (t1/2) and/or PDEs inhibition selectivity. In particular, the $t 1 / 2$ of tadalafil is longer (17-21.6 h) than sildenafil and vardenafil. Furthermore, despite the same high selectivity in inhibiting PDE5, these compounds slightly cross-react with other PDEs, closely related to PDE5 in structure and biochemical properties, to a different extent: sildenafil and vardenafil cross-react with PDE6, and tadalafil with PDE11 (Francis \& Corbin, 2003).

In our previous study, we have demonstrated that tadalafil improved oxidative capacity in $\mathrm{C} 2 \mathrm{C} 12$ skeletal muscle cells in vitro, as displayed by an increased reliance on fat metabolism (Sabatini et al., 2011) accompanied by a better redox status (Duranti et al., 2017).

Interestingly, recent studies have shown that PDE5Is regulate visceral adiposity in a murine diabetic model by shifting adipose tissue cell composition toward a less inflammatory profile, and reduce epicardial adipose tissue in men with type 2 diabetes (Fiore et al., 2016). Furthermore, tadalafil has been shown to reduce the visceral adipose tissue in rabbit (Maneschi et al., 2016) and to improve lean mass content in non-obese men (Aversa et al., 2017). Recently, it has been shown that human fat cell lipolysis can be stimulated through a cGMP-specific pathway, distinct from the one activated by catecholamines (Lafontan et al., 2008). Even though there are many studies, both in vitro and in vivo, reporting various effects of PDE5Is, no study has evaluated the effect of tadalafil on lipolysis in humans.

Starting from this context of knowledge, the purpose of this research was to assess with a double blind randomized study design the impact of a single dose of tadalafil intake on plasma markers of lipolysis in men. We hypothesized that tadalafil administration, through the NO/cGMP pathway, would result in an increase in plasma fatty acids and glycerol concentration in comparison to a placebo group. Elevation in plasma FFAs concentration may cause alterations in the inflammatory responses and insulin resistance (Grant \& Stephens, 2015), hence plasma glucose and cytokines involved in 
Table 1. Subjects' characteristics.

\begin{tabular}{ll}
\hline \multicolumn{2}{l}{ Subjects' characteristics $(\mathrm{n}=12)$} \\
\hline Age (years) & $25.8 \pm 1.1(19-31)$ \\
\hline Height $(\mathrm{cm})$ & $172.6 \pm 2.2(157-184)$ \\
Weight $(\mathrm{kg})$ & $70.6 \pm 1.6(65-84)$ \\
BMI $\left(\mathrm{kg} \cdot \mathrm{m}^{-2}\right)$ & $23.7 \pm 0.4(21.9-26.4)$ \\
\hline
\end{tabular}

BMI, body mass index. Data are expressed as the mean \pm S.E. and respective range of values (min-max).

the inflammatory response, such as interleukin-6 (IL6), tumor necrosis factor- $\alpha$ (TNF $\alpha$ ), interleukin-8 (IL8) and interleukin 10 (IL10) were measured.

\section{METHODS}

Subjects. The study was carried out on blood samples collected from twelve healthy non-smoker male volunteers ( $\mathrm{n}=12$ ) (Table 1) (Di Luigi et al., 2008). All volunteers underwent an endocrinological and sexual history examination conducted by an endocrinologist. The subjects had normal physical and sexual development, functions and followed a standard Mediterranean diet. They were taking no medications, anabolic agents, and/or amino acid supplementation (e.g., arginine). Furthermore, they did not take any dietary supplementations with vitamins and/or antioxidants. Ethical Committee's approval (Policlinico Umberto I - Rome, Italy - prot.n ${ }^{\circ}$ 1039/08) and written informed consent were obtained.

Experimental protocol. After 10 hours of overnight fasting and without caffeine for the prior 24 hours, all of the volunteers reported to the laboratory in the morning hours and randomly received one tablet of placebo or tadalafil per os $\left(20 \mathrm{mg}\right.$, Cialis ${ }^{\circledR}$, Ely-Lilly, Indianapolis, IN, USA) in a double-blind crossover experimental phase. For the placebo trials, the subjects were provided with an identical tablet (except for the presence of tadalafil) and identical instructions (Di Luigi et al., 2008). Then, after a 14-day washout, the same volunteers were crossed over and received either tadalafil or placebo, respectively. Each subject was his own control. No exercise, sexual intercourses, or major stress events were allowed starting from 48 hours before and during the protocol.

Blood sample collection. Blood sample collections (e.g., $10 \mathrm{ml}$ for each draw) were performed immediately before (T0), and at $+2,+6$, and +24 hours after drug/ placebo administration (T2, T6 and T24, respectively). Plasma was immediately separated (3000 $\mathrm{rpm} \times 10 \mathrm{~min}-$ utes, $+4^{\circ} \mathrm{C}$ ) and stored at $-80^{\circ} \mathrm{C}$ until biochemical assays were performed.

Biochemical analysis. All chemical reagents, unless specified otherwise, were purchased from Sigma-Aldrich Chemical (St. Louis, MO, USA).

Glucose. Blood glucose (Glu) was determined spectrophotometrically by a manual procedure using a commercial test kit (Greiner Diagnostic GmbH, Bahlingen-Gremany). Reference values: Glu (70-115 mg/dl).

Free Fatty Acids and Glycerol. FFAs and free Glycerol (Gly) concentrations were determined spectrophotometrically in plasma samples by manual procedures using two commercial test kits (Sigma-Aldrich, St. Louis, MO, USA). Sample ODs were compared according to the
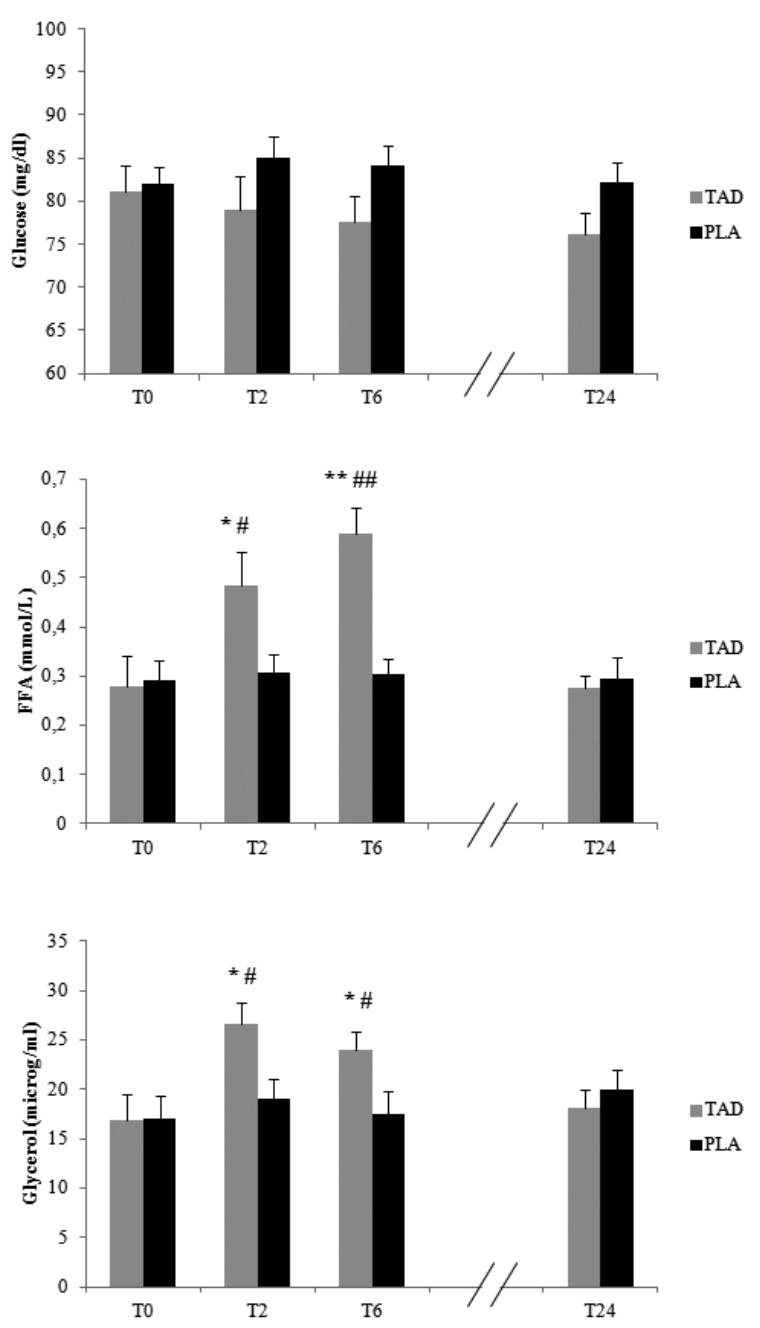

Figure 1. Plasma Glucose, FFAs, and Glycerol analysis.

Glucose, FFAs, and Glycerol levels were measured in plasma before (T0) and 2, 6 and 24 hours (T2-T24) after tadalafil (TAD)/placebo (PLA) administration. Data presented are the mean \pm S.E., $n=12$. ${ }^{*} p<0.05$ and ${ }^{* *} p<0.01$ vs T0, ${ }^{*} p<0.05$ and ${ }^{\# \#} p<0.01$ vs placebo.

manufacturer's recommendations, to those obtained by using Palmitic acid (FFAs) or glycerol standards. Reference values: FFAs (0.1-0.6 mmol/L), Gly (<100 mg/L).

cGMP assay and Total NO content. Plasma cGMP, total NO and nitrate/nitrite levels were measured by an immunoenzymatic and colorimetric assay using commercial kits (R\&D System Inc., Minneapolis, USA) following the manufacturer's recommendations.

Cytokines. Plasma levels of IL6, TNF- $\alpha$, IL8 and IL10 were determined using the Bio-Plex Suspension Array System (Bio-Rad). The limit of sensitivity is 2.6, 1.0, 6.0 and $0.3 \mathrm{pg} / \mathrm{ml}$, and the linear range of detection is 2.3-18880, 5.8-95484, 1.9-26403 and 2.2-8840 pg/ml for IL6, TNF $\alpha$, IL8 and IL10, respectively.

Statistical analysis. The Kolmogorov-Smirnov test was used to evaluate the variable distribution, and all data are expressed as mean values \pm S.E. The SPSS statistical package (Version 21.0 for Windows; SPSS Inc., Chicago, IL, USA) was used for statistical analysis.

A one-way ANOVA for repeated measurements and Bonferroni post-hoc analyses were used to determine significant variations over time and among groups for each parameter evaluated; $p<0.05$ was accepted as significant. 
Table 2. Plasma total NO and cGMP levels.

Total NO, nitrate, nitrite and CGMP plasma levels were measured before (T0) and 2, 6 and 24 hours (T2-T24) after tadalafil administration. Data presented are the mean \pm S.E., $n=12$, the assays were performed in triplicate.

\begin{tabular}{|c|c|c|c|c|c|}
\hline \multicolumn{2}{|c|}{ Variables and groups } & \multirow{2}{*}{$\frac{\text { T0 }}{28.16 \pm 1.62}$} & \multirow{2}{*}{$\frac{\mathrm{T} 2}{24.12 \pm 2.63}$} & \multirow{2}{*}{$\frac{\text { T6 }}{30.20 \pm 4.56}$} & \multirow{2}{*}{$\frac{\text { T24 }}{26.48 \pm 2.80}$} \\
\hline Total NO & TAD & & & & \\
\hline$(\mu \mathrm{mol} / \mathrm{L})$ & PLA & $26.90 \pm 1.72$ & $27.77 \pm 1.44$ & $27.73 \pm 1.78$ & $28.93 \pm 1.93$ \\
\hline Nitrate & TAD & $26.09 \pm 1.57$ & $22.02 \pm 2.61$ & $28.21 \pm 4.44$ & $24.80 \pm 2.77$ \\
\hline$(\mu \mathrm{mol} / \mathrm{L})$ & PLA & $24.90 \pm 1.78$ & $25.67 \pm 1.52$ & $25.44 \pm 1.91$ & $26.81 \pm 2.00$ \\
\hline Nitrite & TAD & $2.07 \pm 0.26$ & $2.10 \pm 0.28$ & $1.99 \pm 0.21$ & $1.68 \pm 0.14$ \\
\hline$(\mu \mathrm{mol} / \mathrm{L})$ & PLA & $2.00 \pm 0.24$ & $2.10 \pm 0.20$ & $2.29 \pm 0.25$ & $2.12 \pm 0.18$ \\
\hline cGMP & $\mathrm{TAD}$ & $202.01 \pm 10.23$ & $198.87 \pm 9.42$ & $198.08 \pm 11.62$ & $199.54 \pm 10.52$ \\
\hline$(\mathrm{pmol} / \mathrm{ml})$ & PLA & $209.49 \pm 9.01$ & $206.76 \pm 11.77$ & $207.96 \pm 9.32$ & $192.14 \pm 14.76$ \\
\hline
\end{tabular}

\section{RESULTS}

Data for plasma glucose, free fatty acids and glycerol levels are presented in Fig. 1. No changes were found in the glucose levels after tadalafil/placebo administration.

Regarding FFAs, a statistically significant group effect was noted $(p=0.00)$, with higher values for tadalafil when compared to the placebo group. A statistically significant group $\times$ time effect was also noted $(p=0.00)$. A maximum increase was found after 6 hours of tadalafil administration $(p<0.01)$; when compared to basal (T0) values, an increase was also found after 2 hours $(p<0.05)$. An interaction effect was noted $(p=0.045)$.

Significant differences were found between tadalafil and placebo groups at 2 hours $(p<0.05)$, and 6 hours post ingestion $(p<0.01) .24$ hours post administration, no differences were found when compared to T0.

Regarding glycerol, higher values were found after 2 hours of tadalafil administration. Compared to T0, the values were found to be significantly higher at 2 and 6 hours $(p<0.05)$. Significant differences were also found between tadalafil and placebo groups at 2 hours $(p<0.05)$, and 6 hours post ingestion $(p<0.05)$.

Data regarding plasma NO and cGMP levels are presented in Table 2. After tadalafil administration, no changes were found in plasma total $\mathrm{NO}$, nitrate, nitrite and cGMP levels when compared to T0 or placebo.

Data for plasma cytokine levels are presented in $\mathrm{Ta}$ ble 3. No statistical significance was found in IL6, IL10 and TNF $\alpha$ plasma levels when compared to T0 or placebo. However, tadalafil significantly decreases IL8 plasma levels after 6 hours of administration when compared to T0 $(12 \%, p<0.05$ vs T0) and to the placebo effect $(15 \%$, $p<0.01$ vs T6 placebo).

\section{DISCUSSION}

The study presented here documents for the first time the impact of an acute oral intake of tadalafil on plasma markers of lipolysis in human subjects. Lipolysis occurs mainly in the adipose tissue and is a catabolic pathway that promotes mobilization of metabolic fuel from the adipose to peripheral tissues to fulfill energy demands. This process involves hydrolysis of triacylglycerols that results in the release of fatty acids and glycerol into the circulation (Saponaro et al., 2015). Our data indicate that tadalafil rapidly increases lipolysis, as evidenced by a significant increase in circulating FFAs and glycerol, 2 and 6 hours post tadalafil administration.

As far as the tadalafil-induced signaling pathway is concerned, it must be considered that PDE5 inhibition enhances the cGMP-dependent endocrine/metabolic effects of NO by further increasing and/or maintaining the intracellular levels of NO-induced cGMP. NO is a signaling molecule that regulates nutrient metabolism; NO stimulates glucose uptake and oxidation, as well as fatty acid oxidation in the insulin-sensitive tissues.

\section{Table 3. Plasma IL6, TNFa, IL10 and IL8 levels}

IL6, TNF $\alpha$, IL10 and IL8 plasma levels were measured before (T0) and 2, 6 and 24 hours (T2-T24) after tadalafil administration. Data presented are the mean \pm S.E., $\mathrm{n}=12$, the assays were performed in triplicate. ${ }^{*} p<0.05$ vs $\mathrm{T} 0,{ }^{*} p<0.01$ s placebo.

\begin{tabular}{lccccc}
\hline Variables and groups & & T0 & T2 & T6 & T24 \\
\hline IL6 & TAD & $5.74 \pm 0.45$ & $6.49 \pm 0.52$ & $5.32 \pm 0.31$ & $5.78 \pm 0.38$ \\
\hdashline$(\mathrm{pg} / \mathrm{ml})$ & PLA & $5.93 \pm 0.27$ & $6.09 \pm 0.34$ & $5.32 \pm 0.27$ & $5.47 \pm 0.28$ \\
\hline TNFa & TAD & $16.44 \pm 1.12$ & $16.05 \pm 1.01$ & $14.48 \pm 0.66$ & $14.87 \pm 0.84$ \\
\hdashline$(\mathrm{pg} / \mathrm{ml})$ & PLA & $13.72 \pm 0.68$ & $14.41 \pm 0.79$ & $12.38 \pm 0.54$ & $13.09 \pm 0.82$ \\
\hline IL10 & TAD & $7.91 \pm 0.67$ & $7.89 \pm 0.67$ & $7.56 \pm 0.54$ & $7.59 \pm 0.72$ \\
\hdashline$(\mathrm{pg} / \mathrm{ml})$ & PLA & $8.36 \pm 0.45$ & $8.29 \pm 0.53$ & $8.39 \pm 0.50$ & $8.05 \pm 0.45$ \\
\hline IL8 & TAD & $5.79 \pm 0.25$ & $6.41 \pm 0.94$ & $5.09 \pm 0.16^{* \#}$ & $5.16 \pm 0.26$ \\
\hline$(\mathrm{pg} / \mathrm{ml})$ & PLA & $6.28 \pm 0.17$ & $6.18 \pm 0.31$ & $5.97 \pm 0.15$ & $5.80 \pm 0.24$ \\
\hline
\end{tabular}


Moreover, NO inhibits the synthesis of glucose, glycogen and fat in the target tissues and enhances lipolysis in adipocytes (Jobgen et al., 2006). Furthermore, it has been reported that PDE5Is may act by increasing NO synthase's activity (Mammi et al., 2011). Most recently, a role of cGMP signaling, as a key regulator of adipogenesis in metabolism and its potential in anti-obesity therapies, has emerged (Moro \& Lafontan, 2013). The measurement of plasma nitrate and nitrite concentrations has been suggested to reflect the endogenous production of $\mathrm{NO}$. Moreover, cGMP has been also measured in plasma as an index of NO production in healthy subjects (Metzger et al., 2006). Indeed, in a model of diabetic mice, chronic treatment with tadalafil enhanced plasma NO oxidation levels (Koka et al., 2014). Noteworthy, in patients treated with a novel long-acting phosphodiesterase 5 inhibitor, a dose-related and continuous increase in plasma cGMP concentration over the 28-day treatment period was observed (Wolk et al., 2009).

Based on this background information, we hypothesized that in human subjects the action of tadalafil would be reflected in the plasma levels of these important signaling molecules.

However, contrary from the above mentioned study, under our experimental conditions, tadalafil did not affect either the plasma cGMP or NO levels, thus indicating that during acute treatment the cGMP and NO plasma levels do not reflect their intracellular increase. Still, we did not perform in vitro experiments which is a limitation of this study; hence, further studies of the PDE5 inhibition performed in tissue samples, especially those obtained from human subjects, would better clarify this issue.

It is well known that the adipose tissue interacts with skeletal muscle, i.e. adipose tissue provides energy-dense lipids to the muscles in order to support the physical exercise. Indeed, it has been demonstrated that PDE5Is take part in the interplay between metabolic processes and skeletal muscle: increases in the lactate release have been reported after PDE5Is treatment in animal muscles and in plasma of athletes after maximum exercise (Di Luigi et al., 2008; Di Luigi et al., 2012).

Previously, we have demonstrated that tadalafil is able to modulate energy homeostasis in the mouse skeletal muscle cells, depending on the treatment length and dose. Supplementation of C2C12 myotubes with tadalafil influences the metabolism by improving FAs oxidation, as displayed by the increase of $3-\mathrm{OH}$ acylCoA dehydrogenase and citrate synthase activities, respectively, which are involved in $\beta$-oxidation and the Krebs cycle (Sabatini et al., 2011). Recently, we have reported that such increase in oxidative metabolism may be beneficial to skeletal muscle cells by enhancing the enzymatic antioxidant system capacity (Duranti et al., 2017).

Since their market output, tadalafil as well as other PDE5 inhibitors have also become popular among men for recreational purpose, particularly in young, healthy populations, as a means of enhancing sexual performance in the absence of ED (Aldridge \& Measham, 1999). When interviewed, they reported that sildenafil enhanced sexual performance by improving sexual desire and producing a "warm" sensation (Bechara et al., 2010), possibly due to vasodilatation and an increase in the energy substrate utilization.

Sexual activity may be considered as an exercise that involves a significant amount of energy expenditure (Frappier et al., 2013). We speculate that the increase in FFAs may also help to support a physical exertion, such as that represented by sexual intercourse; an acute oral intake of tadalafil, in expectation of sexual activity, may provide the energy substrate to fuel skeletal muscles. We can hypothesize that tadalafil, besides increasing FFAs, may also act by favoring their utilization through an increase in the skeletal muscle $\beta$-oxidation, a metabolic pathway that, as occurs in the $\mathrm{C} 2 \mathrm{C} 12$ cells, could be also induced by tadalafil in humans. It is noteworthy that in experiments performed in vitro with the 3T3L1 cells, PDE5 inhibition promoted adipogenesis (Zhang et al., 2010), while in human cultures of fetal skeletal muscle cells tadalafil activated both, the insulin-related signal transduction pathways and the FFA release (Crescioli et al., 2013). Our data show that tadalafil caused a two-fold increase in the free fatty acid level, however, the lack of measurements 6 hours post ingestion may be considered a limitation of the present design; in fact we cannot exclude that a more substantial increase could take place between 6 and 24 hours post ingestion. Moreover, we do not know to what extent the lipid oxidation increases or whether a percentage of the mobilized fatty acids could be re-esterified. We can speculate that in the resting condition, due to the low energy demand, only a small fraction of the fatty acids released from adipocytes would be oxidized. While in the case of a sudden demand for energy, i.e. sexual activity, the increase in FFAs can be useful to quickly match the energy request. Hence, acute lipolysis stimulated by tadalafil may be metabolically healthy, as occurs e.g. in physical exercise; in fact, the recycling of lipids allows cells to free themselves from the potential risk of lipid peroxidation.

An increase in the fatty acids plasma levels can directly or indirectly modify immune and inflammatory responses (Guillherme et al., 2008). Chronically elevated FFAs levels have been shown to cause various detrimental effects in vivo, including impaired insulin sensitivity and inflammation (Sears \& Perry, 2015; Savary et al., 2012).

Also, in the study presented here, plasma levels of IL6, TNF- $\alpha$, IL8, and IL10 involved in inflammatory response were determined. Protective effects of PDE5 inhibitors against inflammation have been reported in diabetic rodents (Venneri et al., 2015). It has been previously shown that tadalafil and vardenafil were able to reduce IL8 in vitro after an inflammatory stimulus (Vignozzi et al., 2013). Interestingly, we found a slight decrease in IL8 plasma levels. On the other hand, IL6, TNF- $\alpha$ and IL10 were unaffected by the acute tadalafil administration. It is noteworthy that in our previous work we have shown that a prolonged exposure to tadalafil also did not affect the IL6 levels, even though it augments oxidative stress and muscle damage (Ceci et al., 2015). In conclusion, our data add a new facet to the pleiotropic actions of PDE5 inhibitors; tadalafil, in human subjects, increases lipolysis as evidenced by a significant increase in circulating FFAs and glycerol that is not accompanied by an inflammatory response. However, our findings are specific to a sample of young, healthy, trained men. Further studies are warranted to determine if similar or more pronounced results can be observed in overweight/sedentary men and to determine if the lipolytic effects extend beyond 6 hours post ingestion. Finally, well-controlled intervention studies are needed to determine the impact of tadalafil administration on the weight/fat loss.

\section{Acknowledgements}

This work was supported by grants from MIUR (PRIN2012-06/2013) and grants from the University of Rome 'Foro Italico' (DIP09-13 and DIP09-14) to SS and SP. Each author of this study declares no relationship 
with the companies or manufacturers who would benefit from the results of the study presented here.

\section{Conflict of interest}

The authors of this article declare no conflicts of interest.

\section{REFERENCES}

Aldridge J, Measham F (1999) Sildenafil (Viagra) is used as a recreational drug in England. Brit Med J 318: 669

Armani A, Marzolla V, Rosano GM, Fabbri A, Caprio M (2011) Phosphodiesterase type 5 (PDE5) in the adipocyte: a novel player in fat metabolism? Trends Endocrinol Metabolism 22: 404-411. doi: 10.1016/j. tem.2011.05.004

Aversa A, Fittipaldi S, Francomano D, Bimonte VM, Greco EA, Crescioli C, Di Luigi L, Lenzi A, Migliaccio S (2017) Tadalafil improves lean mass and endothelial function in nonobese men with mild ED/LUTS: in vivo and in vitro characterization. Endocrine 56: 639-648. doi: 10.1007/s12020-016-1208-y

Aversa A (2010) Systemic and metabolic effects of PDE5-inhibitor drugs. World J Diabet 15: 3-7

Beavo JA, Brunton LL (2002) Cyclic nucleotide research - still expanding after half a century. Nature Rev Mol Cell Biol 3: 710-718

Bechara A, Casabe' A, De Bonis W, Helien A, Bertolino MV (2010) Recreational use of phosphodiesterase type 5 inhibitors by healthy young men. I Sexual Med 7: 3736-3742

Ceci R, Duranti G, Sgrò P, Sansone M, Guidetti L, Baldari C, Sabatini S, Di Luigi L (2015) Effects of tadalafil administration on plasma markers of exercise-induced muscle damage, IL6 and antioxidant status capacity. Eur I Appl Physiol 115: 531-539

Corbin JD, Francis SH (2002) Pharmacology of phosphodiesterase-5 inhibitors. Int J Clin Practice 56: 453-459

Crescioli C, Sturli N, Sottili M, Bonini P, Lenzi A, Di Luigi L (2013) Insulin-like effect of the phosphodiesterase type 5 inhibitor tadalafil onto male human skeletal muscle cells. J Endocrin Invest 36: 1020 1026. doi: $10.3275 / 9034$

Dai Z, Wu Z, Yang Y, Wang J, Satterfield MC, Meininger CJ, Bazer FW, Wu G (2013) Nitric oxide and energy metabolism in mammals. Biofactors 39: 383-391. doi: 10.1002/biof.1099

Di Luigi L, Baldari C, Pigozzi F, Emerenziani GP, Gallotta MC, Iellamo F, Ciminelli E, Sgrò P, Romanelli F, Lenzi A, Guidetti L (2008) The long-acting phosphodiesterase inhibitor tadalafil does not influence athletes' VO2max, aerobic, and anaerobic thresholds in normoxia. Int I Sports Med 29: 110-115

Di Luigi L, Sgrò P, Baldari C, Gallotta MC, Emerenziani GP, Crescioli C, Bianchini S, Romanelli F, Lenzi A, Guidetti L (2012) The phosphodiesterases type 5 inhibitor tadalafil reduces the activation of the hypothalamus-pituitary-adrenal axis in men during cycle ergometric exercise. Am J Physiol Endocrinol Metab 302: E972-E978

Di Luigi L (2008) Supplements and the endocrine system in athletes. Clinics Sports Med 27: 131-151

Duranti G, Ceci R, Sgrò P, Sabatini S, Di Luigi L (2017) Influence of the PDE5 inhibitor tadalafil on redox status and antioxidant defense system in C2C12 skeletal muscle cells. Cell Stress Chaperones 22: 389-396. doi: 10.1007/s12192-017-0778-9

Fiore D, Gianfrilli D, Giannetta E, Galea N, Panio G, di Dato C, Pofi R, Pozza C, Sbardella E, Carbone I, Naro F, Lenzi A, Venneri MA, Isidori AM (2016) PDE5 inhibition ameliorates visceral adiposity targeting the miR-22/SIRT1 pathway: evidence from the CECSID Trial. I Clin Endocrinol Metab 101: 1525-1534

Francis SH, Corbin JD (2003) Molecular mechanisms and pharmacokinetics of phosphodiesterase-5 antagonists. Curr Urol Rep 4: 457-465

Frappier J, Toupin I, Levy JJ, Aubertin-Leheudre M, Karelis AD (2013) Energy expenditure during sexual activity in young healthy couples. PLoS One 8: e79342. doi: 10.1371/journal.pone.0079342
Grant RW, Stephens JM (2015) Fat in flames: influence of cytokines and pattern recognition receptors on adipocyte lipolysis. Am J Physiol Endocrinol Metab 309: E205-E213. doi: 10.1152/ajpendo.00053.2015

Guilherme A, Virbasius JV, Puri V, Czech MP (2008) Adipocyte dysfunctions linking obesity to insulin resistance and type 2 diabetes. Nat Rev Mol Cell Biol 9: 367-377. doi: 10.1038/nrm2391

Jobgen WS, Fried SK, Fu WJ, Meininger CJ, Wu G (2006) Regulatory role for the arginine-nitric oxide pathway in metabolism of energy substrates. J Nutrit Biochem 17: 571-588

Koka S, Aluri HS, Xi L, Lesnefsky EJ, Kukreja RC (2014) Chronic inhibition of phosphodiesterase 5 with tadalafil attenuates mitochondrial dysfunction in type 2 diabetic hearts: potential role of NO/SIRT1/PGC-1 $\alpha$ signaling. Am J Physiol Heart Circul Physiol 306: H1558-H1568

Lafontan M, Moro C, Berlan M, Crampes F, Sengenes C, Galitzky J (2008) Control of lipolysis by natriuretic peptides and cyclic GMP. Trends Endocrinol Metab 19: 130-137. doi: 10.1016/j.tem.2007.11.006

Mammi C, Pastore D, Lombardo MF, Ferrelli F, Caprio M, Consoli C, Tesauro M, Gatta L, Fini M, Federici M, Sbraccia P, Donadel G, Bellia A, Rosano GM, Fabbri A, Lauro D (2011) Sildenafil reduces insulin-resistance in human endothelial cells. PLoS One 6: e14542. doi: 10.1371 /journal.pone.0014542

Maneschi E, Cellai I, Aversa A, Mello T, Filippi S, Comeglio P, Bani D, Guasti D, Sarchielli E, Salvatore G, Morelli A, Mazzanti B, Corcetto F, Corno C, Francomano D, Galli A, Vannelli GB, Lenzi A, Mannucci E, Maggi M, Vignozzi L (2016) Tadalafil reduces visceral adipose tissue accumulation by promoting preadipocytes differentiation towards a metabolically healthy phenotype: Studies in rabbits. Mol Cell Endocrinol 424: 50-70. doi: 10.1016/j.mce.2016.01.015

Metzger IF, Sertorio JT, Tanus-Santos JE (2006) Relationship between systemic nitric oxide metabolites and cyclic GMP in healthy male volunteers. Acta Physiol 188: 123-127

Moro C, Lafontan M (2013) Natriuretic peptides and cGMP signaling control of energy homeostasis. Am J Physiol Heart Circulatory Physiol 304: H358-H368. doi: 10.1152/ajpheart.00704.2012

Sabatini S, Sgrò P, Duranti G, Ceci R, Di Luigi L (2011) Tadalafil alters energy metabolism in $\mathrm{C} 2 \mathrm{C} 12$ skeletal muscle cells. Acta Biochim Polon 58: 237-241

Saponaro C, Gaggini M, Carli F, Gastaldelli A (2015) The subtle balance between lipolysis and lipogenesis: a critical point in metabolic homeostasis. Nutrients 7: 9453-9474. doi: 10.3390/nu7115475

Savary S, Trompier D, Andréoletti P, Le Borgne F, Demarquoy J, Lizard G (2012) Fatty acids - induced lipotoxicity and inflammation. Curr Drug Metab 13: 1358-1370

Sears B, Perry M (2015) The role of fatty acids in insulin resistance. Lipids Health Dis 14: 121. doi: 10.1186/s12944-015-0123-1

Venneri MA, Giannetta E, Panio G, De Gaetano R, Gianfrilli D, Pofi R, Masciarelli S, Fazi F, Pellegrini M, Lenzi A, Naro F, Isidori AM (2015) Chronic Inhibition of PDE5 limits pro-inflammatory monocyte-macrophage polarization in streptozotocin-induced diabetic mice. PLoS One 10: e0126580. doi: 10.1371/journal.pone.0126580

Vignozzi L, Gacci M, Cellai I, Santi R, Corona G, Morelli A, Rastrelli G, Comeglio P, Sebastanelli A, Maneschi E, Nesi G, De Nunzio C, Tubaro A, Mannucci E, Carini M, Maggi M (2013) Fat boosts, while androgen receptor activation counteracts, BPH-associated prostate inflammation. Prostate 73: 789-800. doi: 10.1002/pros.22623

Wolk R, Smith WB, Neutel JM, Rubino J, Xuan D, Mancuso J, Gilbert J, Pressler ML (2009) Blood pressure lowering effects of a new long-acting inhibitor of phosphodiesterase 5 in patients with mild to moderate hypertension. Hypertension 53: 1091-1097. doi: 10.1161/ HYPERTENSIONAHA.109.132225

Zhang X, Ji J, Yan G, Wu J, Sun X, Shen J, Jiang H, Wang H (2010) Sildenafil promotes adipogenesis through a PKG pathway. Biochem Biophys Res Commun 396: 1054-1059. doi: 10.1016/j.bbrc.2010.05.064. 